\title{
Search for a DM annihilation signal from the Galactic Centre halo with H.E.S.S.
}

\author{
Daniil Nekrassov ${ }^{* a}$ and Christopher van Eldik \\ Max-Planck-Institut für Kernphysik, P.O. Box 103980, D 69029 Heidelberg, Germany \\ ${ }^{a}$ E-mail: Daniil.Nekrassov@mpi-hd.mpg.de \\ for the H.E.S.S. Collaboration \\ http://www.mpi-hd.mpg.de/hfm/HESS/
}

The H.E.S.S. experiment, an array of four Imaging Atmospheric Cherenkov Telescopes, observes the Galactic Centre (GC) region in the very high-energy (VHE, E > $100 \mathrm{GeV}$ ) $\gamma$-ray domain since 2003. The GC is believed to be the region with the highest Dark Matter (DM) density in our Galaxy, thus making it one of the primary targets to look for photons from annihilation or decay of DM particles. The interpretation of the collected H.E.S.S. data with regard to possible DM signals is however complicated by the presence of several astrophysical sources of VHE $\gamma$-ray radiation in this region. A new analysis of archival H.E.S.S. data from the GC region avoids such contamination by searching for a DM signal towards a region of projected distance $r \sim 45-150$ pc from the GC. No residual $\gamma$-ray emission is found in the energy range between $300 \mathrm{GeV}$ and $30 \mathrm{TeV}$, leading to stringent upper limits on the velocity-weighted DM annihilation cross section $\langle\sigma v\rangle$. In particular, values for $\langle\sigma v\rangle$ above $3 \times 10^{-25} \mathrm{~cm}^{3} \mathrm{~s}^{-1}$ are excluded for the Einasto density profile parametrisation.

25th Texas Symposium on Relativistic Astrophysics - TEXAS 2010

December 06-10, 2010

Heidelberg, Germany

* Speaker. 


\section{Introduction}

High-energy $(100 \mathrm{MeV}-100 \mathrm{GeV})$ and very high-energy $\gamma$-ray observations are a promising way to search for signals from interactions of DM particles in space. Viable particle DM candidates are WIMPs (Weakly Interacting Massive Particles), which are provided in various extensions of the standard model (SM) of particle physics, like Supersymmetry (SUSY) (see, e.g., [1] and references therein). The self-annihilation (or decay) of such WIMPs would create SM particles in the final state, including $\gamma$-rays, produced mostly in hadronization processes. Since the annihilation spectrum of $\gamma$-rays extends up to the WIMP mass, which is expected to be in the range between $10 \mathrm{GeV}$ and several TeV, DM annihilation (or decay) might be detectable at high and very high energies. Targets of predicted large DM energy density, which have been observed by either Fermi-LAT or ground-based $\gamma$-ray instruments include dwarf galaxies $[2,3,4,5,6]$, intermediate mass black holes [7], and the Galactic Centre (GC) [8].

For the case of DM self-annihilation, the expected $\gamma$-ray flux $\Phi$ from an observed sky region of angular size $\Delta \Omega$ can be computed as follows:

$$
\frac{d \Phi\left(\Delta \Omega, E_{\gamma}\right)}{d E_{\gamma}}=\frac{1}{4 \pi} \frac{\langle\sigma v\rangle}{\mathrm{m}_{\chi}^{2}} \frac{d N}{d E_{\gamma}} \times \bar{J} \Delta \Omega, \text { where } \bar{J}=\frac{1}{\Delta \Omega} \int_{\Delta \Omega} \int_{\text {line-of-sight }} \rho^{2}(r) d s .
$$

Here, $\langle\sigma v\rangle$ is the velocity-weighted annihilation cross-section of the corresponding dark matter (DM) candidate of mass $m_{\chi}$, and $\frac{d N}{d E_{\gamma}}$ is the average spectrum of $\gamma$-rays produced in the annihilation/hadronisation process. Note that these terms solely depend on the assumed particle physics model. The astrophysical part of the equation is represented by $\bar{J}$, which is the averaged line-ofsight integral of the squared DM density $\rho(r)$. The latter term depends on the selected target of observation. Here we concentrate on the GC, which is a very promising region both due to its proximity and a presumably high DM density (see [9], [10] for results from recent N-body simulations).

The analyses presented here were carried out using GC data from H.E.S.S., an array of four Imaging Atmospheric Cherenkov Telescopes (IACTs), located in the Khomas Highlands of Namibia, which regularly observes the GC since 2003. The deep exposure of this region revealed several sources of VHE $\gamma$-rays, all of which can be explained to date by conventional astrophysical processes. In particular, the detection of the point source HESS J1745-290 [11], positionally coincident with the supermassive black hole Sgr A* located in the dynamical centre of the Galaxy, raised a lot of attention with regard to DM interactions as a possible origin of the emission. However, DM interactions could be convincingly ruled out as a main contribution to the observed emission [8], and upper limits on the velocity-weighted annihilation cross section $\langle\sigma v\rangle$ were derived $[12,13]$. Note that these limits are subject to large systematic uncertainties, both due to the unknown shape on the DM density profile in the very centre of the Galaxiy and the presence of astrophysical background processes. Furthermore, a band of extended $\gamma$-ray emission detected along the GC ridge [14] was also used to derive upper limits on a DM induced signal [17, 18]. These limits put tight constraints on leptophilic DM models, which were brought into play to explain the recent electron/positron measurements by Pamela [15] and Fermi [16]. 


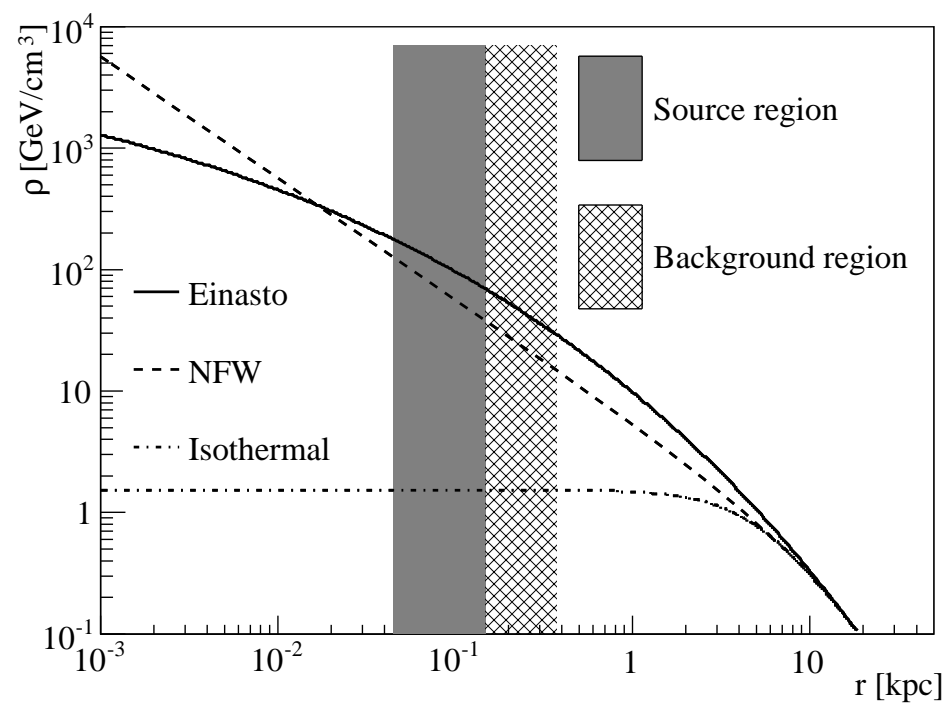

Figure 1: Comparison of the Galactic DM halo profiles used in this analysis. The parameters for the NFW and Einasto profiles are taken from [20]. An isothermal profile [1], exhibiting a flat DM density out to a galactocentric distance of $1 \mathrm{kpc}$, is shown for comparison. All profiles are normalized to the local DM density $\left(\rho_{0}=0.39 \mathrm{GeV} / \mathrm{cm}^{3}[20]\right.$ at a distance of $8.5 \mathrm{kpc}$ from the GC). The source region and the region used for background estimation are indicated. Note that the predicted DM density is always larger in the source region, except for the isothermal profile, which is included for completeness.

\section{Search for DM signals from the GC halo region}

As discussed in the previous section, several astrophysical processes take place in the GC region which produce VHE $\gamma$-rays which are a significant background for a search for DM signals. To circumvent this problem, areas of the GC region free of astrophysical $\gamma$-ray sources, i.e. above and below the Galactic plane, were studied. On the one hand, this means that the very centre, where the DM density is the largest, has to be avoided. On the other hand, systematic uncertainties due to the presence of astrophysical background and due to the unknown shape of the DM density profile in the very centre are reduced. Besides, it can be expected that such an analysis would improve the results achieved on the diffuse emission region [17].

For the analysis [19], more than 100 hours of archival H.E.S.S. observations were used. $\gamma$-rays from DM annihilations were searched for in a circular source region of radius $R_{\mathrm{on}}=1.0^{\circ}$ centered at the GC. Contamination of the DM signal by local astrophysical $\gamma$-ray sources was excluded by restricting the analysis to Galactic latitudes $|b|>0.3^{\circ}$. A new technique was developed to estimate the $\gamma$-ray-like hadronic background from the same FoV, called the reflected pixel method. It allows to make efficient use of the archival data and obtain a robust estimation of the background in the source region. Since the background was estimated from areas outside the $1^{\circ}$ source region, the analysis remains sensitive to a potential DM signal for density profiles monotonously falling off as a function of the galactocentric distance (see Fig. 1). 
The $\gamma$-ray spectrum derived from the source region was found to be in full agreement with a null measurement after subtraction of the background spectrum. Thus upper limits on $\langle\sigma v\rangle$ were calculated as a function of the DM particle mass (see Fig. 2). To estimate the DM density along the line of sight, the NFW and Einasto profile parametrisations shown in Fig. 1 were used [20], yielding upper limits which are among the best for VHE energies so far, and reaching $3 \times 10^{-25} \mathrm{~cm}^{3} \mathrm{~s}^{-1}$ for $m_{\chi} \approx 1 \mathrm{TeV}$ and the Einasto density profile. Besides, the limits differ only a factor of two between both halo profile parametrisations. Therefore, this analysis is less sensitive to the actual DM profile shape compared to observations of the very centres of galaxies.

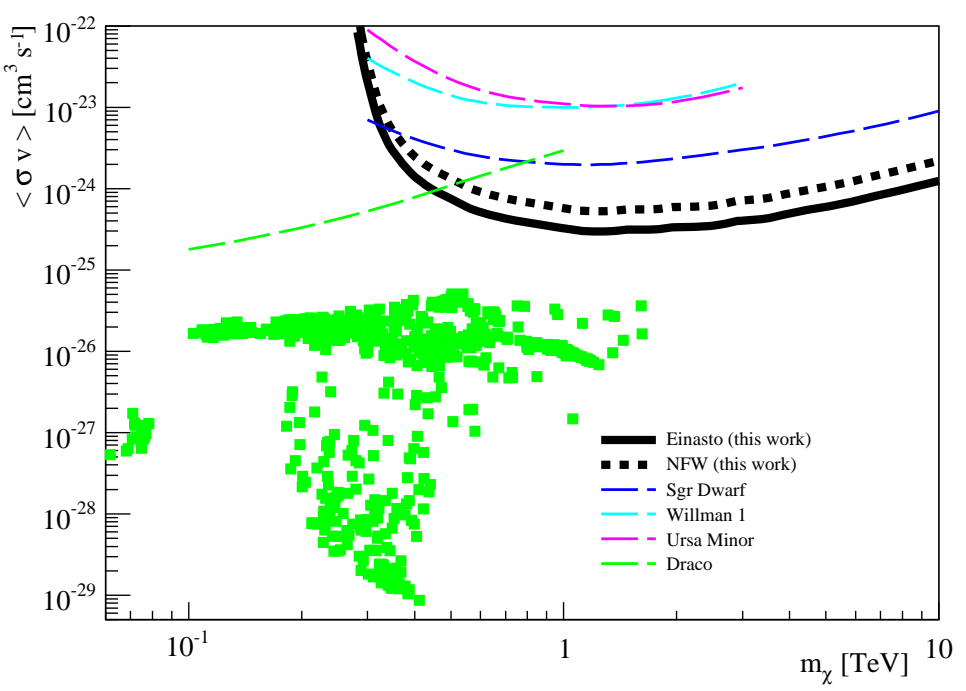

Figure 2: Upper limits (at 95\% CL) on the velocity-weighted annihilation cross-section $\langle\sigma v\rangle$ as a function of the DM particle mass $m_{\chi}$ for the Einasto and NFW density profiles. The best sensitivity is achieved at $m_{\chi} \sim 1 \mathrm{TeV}$. For comparison, the best limits derived from observations of dwarf galaxies at very high energies, i.e. Sgr Dwarf [2], Willman 1, Ursa Minor [5] and Draco [4], using in all cases NFW shaped DM profiles, are shown. Similar to the sky region investigated in the presented analysis, dwarf galaxies are objects free of astrophysical background sources. The green points represent DarkSUSY models [21], which are in agreement with WMAP and collider constraints and were obtained with a random scan of the mSUGRA parameter space using the following parameter ranges: $10 \mathrm{GeV}<M_{0}<1000 \mathrm{GeV}, 10 \mathrm{GeV}<$ $M_{1 / 2}<1000 \mathrm{GeV}, A_{0}=0,0<\tan \beta<60, \operatorname{sgn}(\mu)= \pm 1$.

\section{Conclusions}

The GC region remains one of the most promising targets for the indirect search for DM due to its proximity and a presumably high density of DM particles. However, in the VHE $\gamma$-ray domain, astrophysical $\gamma$-ray radiation hampers the search for DM signals. An improvement upon previous results was achieved by analysing archival H.E.S.S. data in region free of astrophysical background, yielding sensitive contraints on the velocity-weighted DM annihilation cross-section $\langle\sigma v\rangle$. At the same time, uncertainties due to the shape of the DM density profile were substantially reduced. 


\section{References}

[1] G. Bertone et al., Particle dark matter: evidence, candidates and constraints, Phys. Rep., 405 (2005) 279 [hep-ph / 0404175$]$

[2] F. Aharonian et al., Observations of the Sagittarius dwarf galaxy by the HESS experiment and search for a dark matter signal,

Astrop. Phys., 29 (2008) 55 [astro-ph/0711.2369]

[3] F. Aharonian et al, A Search for a Dark Matter Annihilation Signal Toward the Canis Major Overdensity with H.E.S.S.,

Astrophys. J., 691 (2009) 175 [arXiv:0809.3894]

[4] A. A. Abdo et al., Observations of Milky Way Dwarf Spheroidal Galaxies with the Fermi-Large Area Telescope Detector and Constraints on Dark Matter Models,

Astrophys. J., 712 (2010) 147 [astro-ph/1001 . 4531]

[5] V. A. Acciari et al., VERITAS Search for VHE Gamma-ray Emission from Dwarf Spheroidal Galaxies , Astrophys. J., 720 (2010) 1174 [astro-ph/1006.5955]

[6] A. Abramowski et al, H.E.S.S. constraints on Dark Matter annihilations towards the Sculptor and Carina Dwarf Galaxies, Astrop. Phys., 34 (2011) 608 [arXiv: 1012.5602$]$

[7] F. Aharonian et al, Search for gamma rays from dark matter annihilations around intermediate mass black holes with the HESS experiment,

Phys. Rev., D78 (2008) 072008 [arXiv: 0806.2981$]$

[8] F. Aharonian et al., HESS Observations of the Galactic Center Region and Their Possible Dark Matter Interpretation, Phys. Rev. Lett., 97 (2006) 221102 [astro-ph/ 0610509 ]

[9] V. Springel et al., Prospects for detecting supersymmetric dark matter in the Galactic halo, Nature, 456 (2008) 73

[10] J. Diemand et al., Clumps and streams in the local dark matter distribution, Nature, 454 (2008) 735

[11] F. Aharonian et al., Very high energy gamma rays from the direction of Sagittarius $A^{*}$, Astron. Astrophys., 425 (2004) L13 [astro-ph/ 0406658 ]

[12] J. Ripken et al., Update on Dark Matter in the Galactic Centre with H.E.S.S., in proceedings of ICRC 09

[13] J. Ripken et al., Direct constraints on the CMSSM using H.E.S.S. observations of the Galactic Centre and the Sagittarius dwarf galaxy, [astro-ph/1012 . 3939]

[14] F. Aharonian et al., Discovery of very-high-energy $\gamma$-rays from the Galactic Centre ridge, Nature, 439 (2006) 695 [astro-ph/ 0603021$]$

[15] O. Adriani et al., An anomalous positron abundance in cosmic rays with energies 1.5-100GeV, Nature, 458 (2008) 607 [astro-ph / 0810 . 4995]

[16] M. Ackermann et al., Fermi LAT observations of cosmic-ray electrons from 7 GeV to $1 \mathrm{TeV}$, Phys. Rev., D82 (2010) 092004, astro-ph/1008 . 3999

[17] P. Meade et al., Dark Matter interpretations of the excesses after FERMI, Nuclear Physics B, 831 (2010) 178 [hep-ph/0905.0480] 
[18] R. M. Crocker et al., Radio and gamma-ray constraints on dark matter annihilation in the Galactic center, Physical Review D, 81 (2008) 63516 [hep-ph/1002 . 0229]

[19] A. Abramowski et al., Search for a Dark Matter annihilation signal from the Galactic Center halo with H.E.S.S., Phys. Rev. Lett., in press, [astro-ph/1103.3266]

[20] L. Pieri et al, Implications of High-Resolution Simulations on Indirect Dark Matter Searches, [astro-ph/0908.0195]

[21] P. Gondolo et al, DarkSUSY: computing supersymmetric dark matter properties numerically, J. Cosm. Astrop. Phys., 7 (2004) 8, [http: //www.physto.se/ eds jo/darksusy/] 